\title{
Wavelength tuning of femtosecond pulses generated in nonlinear crystals by using diffractive lenses
}

\author{
G. Mínguez-Vega, ${ }^{1,2, *}$ C. Romero, ${ }^{3}$ O. Mendoza-Yero, ${ }^{1,2}$ J. R. Vázquez de Aldana, ${ }^{3}$ R. Borrego-Varillas, ${ }^{3}$ \\ C. Méndez, ${ }^{4}$ P. Andrés, ${ }^{5}$ J. Lancis, ${ }^{1,2}$ V. Climent, ${ }^{1,2}$ and L. Roso ${ }^{4}$ \\ ${ }^{1}$ GROC•UJI, Departament de Física, Universitat Jaume I, 12080 Castelló, Spain \\ ${ }^{2}$ Institut de Noves Tecnologíes de la Imatge, Universitat Jaume I, 12080 Castelló, Spain \\ ${ }^{3}$ Departamento de Física Aplicada, Universidad de Salamanca, E-37008 Salamanca, Spain \\ ${ }^{4}$ Centro de Láseres Pulsados Ultracortos Ultraintensos, E-37008 Salamanca, Spain \\ ${ }^{5}$ Departament d'Òptica, Universitat de València, E-46100 Burjassot, Spain \\ *Corresponding author: gminguez@uji.es
}

Received June 25, 2010; revised September 14, 2010; accepted October 7, 2010;

posted October 11, 2010 (Doc. ID 130689); published October 28, 2010

We demonstrate that diffractive lenses (DLs) can be used as a simple method to tune the central wavelength of femtosecond pulses generated from second-order nonlinear optical processes in birefringent crystals. The wavelength tunability is achieved by changing the relative distance between the nonlinear crystal and the DL, which acts in a focusing configuration. Besides the many practical applications of the so-generated pulses, the proposed method might be extended to other wavelength ranges by demonstrated similar effects on other nonlinear processes, such as high-order harmonic generation. (C) 2010 Optical Society of America

OCIS codes: $\quad 050.0050,120.6200,190.4410,190.7110$.

The generation of nonlinear effects with high efficiency and flexibility has a clear implication for many applications, such as high-resolution optical microscopy [1], petawatt-class laser development via optical parametric amplification [2], or industrial pattern recognition [3]. For ultrafast optics, diffractive elements play a significant role in tailoring nonlinear processes, because they allow us to modify the spatial and temporal shape of the pulse. For example, diffractive gratings recorded in a birefringent crystal increase the efficiency of second-order nonlinear processes [4]. Moreover, Fresnel diffraction through circular apertures enhances supercontinuum generation by exploiting the lensing effect [5].

One of the most common diffractive optical elements is a diffractive lens (DL). This optical element focuses light by diffraction. The well-known Fresnel zone plate can be regarded as a poor diffraction efficiency DL. In experiments with nanosecond pulses, the Fresnel zone plate has demonstrated its usefulness to enhance the efficiency of third-harmonic (TH) generation up to a factor of 16 [6]. However, to the best of our knowledge, no attention has been paid to the basic problem of the generation of second-order nonlinear processes with DLs and femtosecond pulses. In this case, the large spectral bandwidth of the pulsed light and the strong dependence of diffraction on the wavelength cause new observable facts.

In this Letter, we experimentally study nonlinear frequency-conversion processes [7] of ultrashort pulses focused by a kinoform DL in nonlinear crystals. A kinoform DL is a phase DL that provides the highest diffraction efficiency [8]. Wavelength tuning of both second-harmonic ( $\mathrm{SH})$ generation and sum-frequency generation is demonstrated. The proposed method provides a novel technique to tune the central wavelength of the generated pulses with potential applications to other nonlinear processes.
Within the paraxial approximation, the generation of nonlinear effects by focusing a $\mathrm{cw}$ beam with refractive or DLs of the same NA is expected to show similar behavior. However, for a femtosecond pulse, strong differences appear owing to the large spectral bandwidth of pulsed radiation. In this case, the intensity distribution in the focal region of a refractive lens is different from that of a DL. Chromatic aberrations are the cornerstone of the above behavior. In particular, for a refractive lens, chromatic aberrations are caused by material dispersion [9], whereas, for a DL, they are due to the diffraction phenomenon [10]. The focal length of the $\mathrm{DL}, f$, is related to the wavelength, $\lambda$, by the expression $\lambda f=\lambda_{a} f_{o}$, where the subscript $o$ denotes the reference wavelength. For a DL made up of a low-dispersion material, the axial extension of the focal region is given by $\Delta f=f_{o} \Delta \lambda / \lambda_{o}$, where $\Delta \lambda$ is the bandwidth of the light source. This is several orders of magnitude larger than the focal region achieved with a singlet having a refractive index $n, \Delta f=-f_{o} \Delta \lambda(d n /$ $d \lambda)_{o} / n-1$. For an achromatic doublet, the focal region in the axial direction is the lowest due to the lack of chromatic aberrations. Note that the different spectral and spatial geometry of the focal volume will essentially affect the processes of frequency conversion induced with each lens.

The whole experimental setup is depicted in Fig. 1 . The laser system was a Ti:sapphire regenerative amplifier (Spitfire, Spectra-Physics) that operates at a $1 \mathrm{kHz}$ repetition rate, delivering pulses of $\tau_{F}=120 \mathrm{fs}$ FWHM, and a central wavelength of $795 \mathrm{~nm}$. The $1 / e^{2}$ diameter of the beam is about $9 \mathrm{~mm}$. A sample of the beam is taken for the SH generation experiment (dotted box) and is focused by a kinoform DL (Institute of Automatics and Electrometry, Russia) with a focal length of $150 \mathrm{~mm}$, for the design wavelength of $565 \mathrm{~nm}$. In our experiment, the focal distance for the wavelength of $795 \mathrm{~nm}$ corresponds to $106.6 \mathrm{~mm}$. The DL was placed in a computer- 


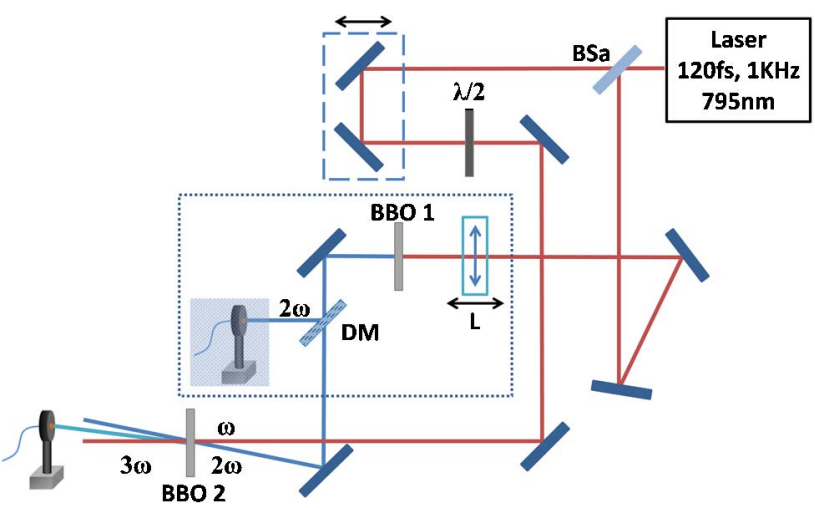

Fig. 1. (Color online) Experimental setup: BSa, beam sampler (10/90); $\lambda / 2$, half-wave plate; BBO, nonlinear crystal; and L, diffractive/refractive lens mounted on a motorized stage.

controlled motorized stage that moves the lens along the propagation axis. Near the focal region, an uncoated Type-I $\beta$ - $\mathrm{BaB}_{2} \mathrm{O}_{4} \quad(\mathrm{BBO})$ crystal $(10 \mathrm{~mm} \times 10 \mathrm{~mm} \times$ $\left.0.5 \mathrm{~mm}, \theta=29.1^{\circ}, \varphi=0^{\circ}\right)$, denoted by BBO1 in Fig. 1 , was aligned at quasi-normal incidence. Initially the BBO1 crystal was located at a distance $z=104 \mathrm{~mm}$ from the DL, and then this distance was increased with the motorized stage by steps of $75 \mu \mathrm{m}$. We captured the spectrum of the SH pulses, after reflecting at the dichroic mirror (DM), with a spectrometer (HR4000, Ocean Optics). The trace of the SH spectrum versus crystal-lens distance is shown in Fig. 2(a). For comparison, we also performed the above experiment replacing the DL by an achromatic doublet with a focal length of $100 \mathrm{~mm}$ (Linos G063144525), in order to get approximately the same NA. Results are shown in Fig. 2(b).

From Fig. 2, one can see that using the DL, the central wavelength of the $\mathrm{SH}$ pulse is tuned. Whenever the axial position of the BBO crystal coalesces with the focal position of the DL for a certain wavelength $\lambda$, then the central wavelength of the generated $\mathrm{SH}$ signal turns out to be
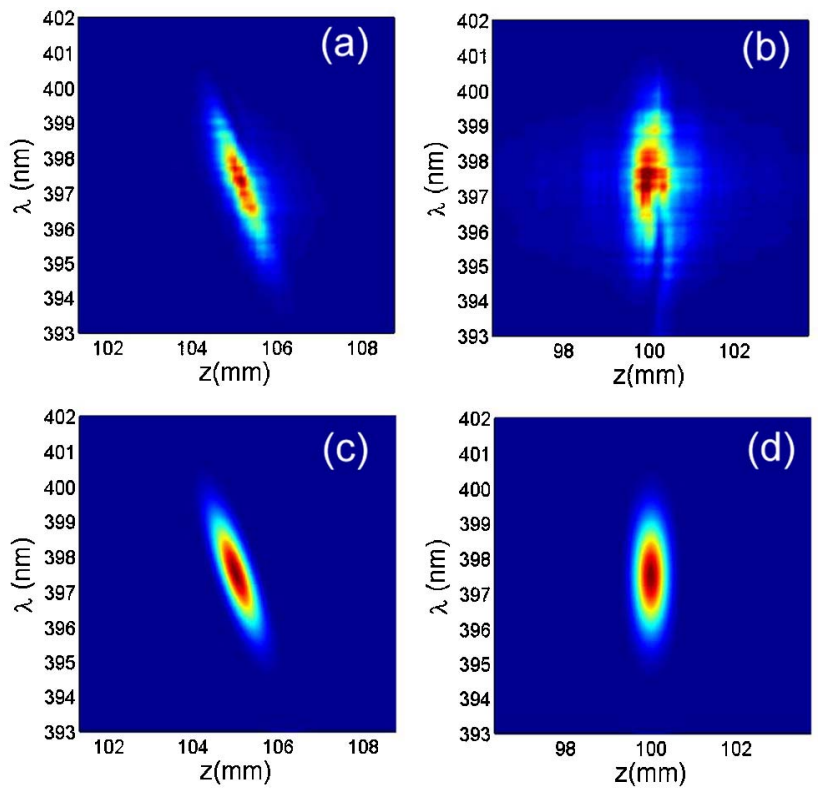

Fig. 2. (Color online) Measured SH spectrum versus the lenscrystal (BBO1) distance around the focal length of (a) a kinoform DL and (b) an achromatic doublet. (c) and (d) correspond to numerical simulations of the traces. $\lambda / 2$. Of course, the tunability range depends on both the spectrum of the incident pulse and the phase-matching spectral acceptance of the crystal. In our experiment, the crystal thickness is short enough to avoid limitation due to phase matching.

To simulate the above results, we use a very simple model based on the thin nonlinear crystal approximation with undepleted pump. We consider that a plane wave illuminates an ideal refractive or DL with $9 \mathrm{~mm}$ of aperture. The spectrum of the laser was modeled as a Gaussian spectrum. The power spectrum of the generated SH for each distance crystal-lens distance $z$ can be roughly estimated by following the well-known expression $I(\lambda / 2, z) \propto I^{2}(\lambda, z)\left[\sin \left(\Delta k_{\lambda} \cdot L / 2\right) /\left(\Delta k_{\lambda} \cdot L / 2\right)\right]^{2}$ [7], where $I(\lambda, z)$ is the power spectrum of the fundamental beam in each $z$ position, $L$ is the crystal length, and $\Delta k_{\lambda}$ is the phase mismatch corresponding to the BBO crystal at normal incidence. In the simulation, we can consider the normal incidence approximation because the confocal parameter is greater than the crystal length. Results of these calculations are depicted in Figs. 2(c) and 2(d), showing very good agreement with experiments. It should be mentioned that a more accurate formulation can be used to simulate the experimental results; however, this simple approximation is enough to show the dominant effects.

To avoid damage of the crystal, the fundamental pulse energy was set in all cases to $44 \mathrm{~nJ}$. SH conversion efficiency was measured for the two lenses at the crystallens distance that maximized the output energy. We obtained $25 \%$ and $10 \%$ of total efficiency with the refractive and the DL, respectively. The confocal parameter of the refractive lens $(b=250 \mu \mathrm{m})$ would require a larger crystal thickness to optimize the maximum efficiency condition for focused beams, $L=2.84 \cdot b$ [7], but with such a choice, the phase-matching spectral efficiency would limit the converted spectrum. For the $\mathrm{SH}$ with a DL, the reduction in efficiency is mainly caused by two factors. The first factor is the spatial distribution of the spectrum that avoids the conversion of all the wavelengths at the same position of the crystal. The second factor is the limited diffraction efficiency of our DL for the central wavelength of the laser (about 69\%). This efficiency can be improved with an optimal design of the DL for the desired wavelength. Then the efficiency of the SH generation with the DL would be close to the one obtained with the refractive lens.

From Fig. 2 we can also realize that the DL acts as a bandpass optical filter, reducing the SH spectral width. In particular, the spectral width of the $\mathrm{SH}$ pulses is $1.5 \mathrm{~nm}$ for the pulses generated with the DL and $2.4 \mathrm{~nm}$ for those generated with the achromat. This wavelength clipping reduces the well-known angular dispersion of the $\mathrm{SH}$ generated with focusing optics. Because of this fact, we did not introduce any correcting system. Moreover, an enlargement of the pulse duration is expected for such bandwidth reduction. Assuming a transform-limited pulse, the temporal duration of a SH pulse with $1.5 \mathrm{~nm}$ spectral width is $145 \mathrm{fs}$. The temporal characterization of the pulse was carried out by the cross correlation of the $\mathrm{SH}$ with the fundamental pulse. The setup is shown in Fig. 1. For these measurements, the DM was removed and the crystal-DL distance was fixed at the position of 

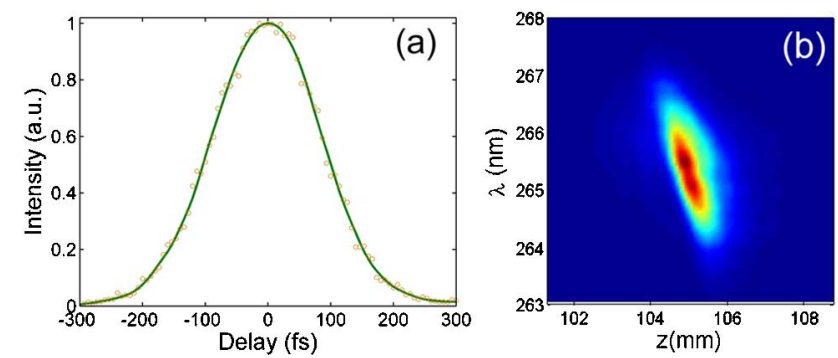

Fig. 3. (Color online) Experimental measurements: (a) crosscorrelation trace of the SH generated with the kinoform DL and (b) TH spectrum versus the lens-crystal (BBO1) distance around the focal length of the kinoform DL.

maximum SH signal. The fundamental and the $\mathrm{SH}$ were mixed in a $100 \mu \mathrm{m}$ Type I BBO crystal (BBO2 in the figure, $6 \mathrm{~mm} \times 6 \mathrm{~mm} \times 0.1 \mathrm{~mm}, \theta=44.3^{\circ}, \varphi=90^{\circ}$ ), producing a TH signal whose power was measured in terms of the relative delay. The width of the recorded trace [depicted in Fig. 3(a)] was 200 fs. Such temporal width suggests [11] a duration of the $\mathrm{SH}$ pulses of $\sim 150 \mathrm{fs}$ (assuming an enlargement of the fundamental to $130 \mathrm{fs}$ at $\mathrm{BBO} 2$ ), which is in fairly good agreement with the transform-limited pulse duration of the measured $\mathrm{SH}$ spectra.

Finally, we demonstrated the wavelength tuning of the TH signal by using the experimental setup for the crosscorrelation measurements. The SH pulses were generated by focusing the beam in BBO1 and then mixed in $\mathrm{BBO} 2$ with the fundamental unfocused beam. To reduce the angle between the two beams, the last mirror was replaced by a DM. Next, the delay line length was fixed to maximize the TH signal and again the tunability was obtained by changing the distance between the DL and $\mathrm{BBO}$. The trace of the $\mathrm{TH}$ spectrum versus the DL-BBO1 distance is shown in Fig. 3(b). The TH signal can be tuned, thanks to the wavelength selection induced in the SH generation by means of the DL.

In conclusion, we have experimentally demonstrated a compact and robust technique using a DL that is able to achieve wavelength tunability in femtosecond pulses generated from second-order nonlinear optical processes by simply changing the DL-crystal length. An alternative technique to produce a similar tunability could be achieved by changing the orientation of a thick nonlinear crystal where the nonlinear process is taking place. However this choice is not suitable for ultrashort pulses because the shifting of the phase-matching curve in relation to the pulse spectrum will lead to the generation of a modulated spectrum in the SH pulse and therefore a degraded temporal shape. In addition to the direct application of the generated tunable pulses to experiments in spectroscopy, the proposed technique can be extended to other processes like optical parametric amplification or even to high-order harmonic generation.

We acknowledge support from the Spanish Ministerio de Ciencia e Innovación through the Consolider Program SAUUL CSD2007-00013, research projects FIS2009-09522 and FIS2010-15746, and Junta de Castilla y León through the Program for Groups of Excellence (GR27). We also acknowledge support from the Centro de Láseres Pulsados.

\section{References}

1. E. Papagiakoumou, V. de Sars, D. Oron, and V. Emiliani, Opt. Express 16, 22039 (2008).

2. I. N. Ross, P. Matousek, G. H. C. New, and K. Osvay, J. Opt. Soc. Am. B 19, 2945 (2002).

3. S.-Y. Tseng, C. Fuentes-Hernandez, and B. Kippelen, Opt. Lett. 32, 2599 (2007).

4. C. Romero, J. R. Vázquez de Aldana, C. Méndez, and L. Roso, Opt. Express 16, 18109 (2008).

5. X. Ni, C. Wang, X. Liang, M. AL-Rubaiee, and R. R. Alfano, IEEE J. Select. Top. Quantum Electron. 10, 1229 (2004).

6. S. Cavalieri, L. Fini, E. Sali, and R. Buffa, Opt. Lett. 31, 1298 (2006).

7. R. W. Boyd, Nonlinear Optics (Academic, 2008).

8. V. Moreno, J. F. Román, and J. R. Salgueiro, Am. J. Phys. 65, 556 (1997).

9. Z. Bor, J. Mod. Opt. 35, 1907 (1988).

10. R. Ashman and M. Gu, Appl. Opt. 42, 1852 (2003).

11. A. Fürbach, T. Le, S. Spielmann, and F. Krausz, Appl. Phys. B 70, s37 (2000). 